\title{
Mixed-mode pattern in Doublefoot mutant mouse limb-Turing reaction-diffusion model on a growing domain during limb development
}

\author{
Takashi Miura $^{\mathrm{a}, *}$, Kohei Shiota ${ }^{\mathrm{b}}$, Gillian Morriss-Kay ${ }^{\mathrm{c}}$, Philip K. Maini ${ }^{\mathrm{d}}$ \\ ${ }^{a}$ Congenital Anomaly Research Center, Kyoto University Graduate School of Medicine, Yoshida Konoe-chou, Sakyo-Ku, 606-8501, Japan \\ ${ }^{\mathrm{b}}$ Department of Anatomy and Developmental Biology, Kyoto University Graduate School of Medicine, Japan \\ ${ }^{\mathrm{c}}$ Department of Human Anatomy and Genetics, University of Oxford, South Parks road, Oxford, OX1 3QX, UK \\ ${ }^{\mathrm{d}}$ Centre for Mathematical Biology, University of Oxford, 24-29 St. Giles, Oxford, OX1 3LB, UK
}

Received 21 June 2005; received in revised form 24 October 2005; accepted 25 October 2005

Available online 20 December 2005

\begin{abstract}
It has been suggested that the Turing reaction-diffusion model on a growing domain is applicable during limb development, but experimental evidence for this hypothesis has been lacking. In the present study, we found that in Doublefoot mutant mice, which have supernumerary digits due to overexpansion of the limb bud, thin digits exist in the proximal part of the hand or foot, which sometimes become normal abruptly at the distal part. We found that exactly the same behaviour can be reproduced by numerical simulation of the simplest possible Turing reaction-diffusion model on a growing domain. We analytically showed that this pattern is related to the saturation of activator kinetics in the model. Furthermore, we showed that a number of experimentally observed phenomena in this system can be explained within the context of a Turing reaction-diffusion model. Finally, we make some experimentally testable predictions.
\end{abstract}

(C) 2005 Elsevier Ltd. All rights reserved.

Keywords: Limb development; Turing reaction-diffusion; Mixed-mode

\section{Introduction}

Periodic pattern formation occurs at various stages and locations during development. Formation of periodic structure can be seen in skin pigment pattern (Bard, 1981; Kondo and Asai, 1995), feather follicle formation (Jung et al., 1998), digit formation during development (Newman and Frisch, 1979; Miura and Shiota, 2000b) and so forth. These patterns cannot simply be explained by positional information theory and some developmental biologists are beginning to utilize the Turing reactiondiffusion model (Turing, 1952) to understand the mechan-

\footnotetext{
*Corresponding author. Tel.: + 8175753 4345; fax: + 81757534621 .

E-mail addresses: miura-takashi@umin.ac.jp (T. Miura), shiota@anat1.med.kyoto-u.ac.jp (K. Shiota), gillian.morriss-kay@anat.ox.ac.uk (G. Morriss-Kay), maini@maths.ox.ac.uk (P.K. Maini).
}

ism of biological pattern formation (Gilbert, 2004; Wolpert, 1998).

One of the most extensively studied periodic patterning systems is digit formation during limb development (Maini and Solursh, 1991). In 1979 it was postulated that a Turing-type reaction-diffusion system could account for limb pattern formation, and the model reproduced the increase in the number of chondrogenic loci in the distal part of the limb bud (Newman and Frisch, 1979; see also Othmer, 1986; Newman et al., 1988). The Turing reaction-diffusion model is one of the class of activator-inhibitor models (Gierer and Meinhardt, 1972) and in fact gives patterns very similar to the later proposed mechanochemical models of Oster and Murray (Oster et al., 1983). This work has been neglected by developmental biologists since molecular biology became the predominant approach, but has remained influential in the field of mathematical biology (Murray, 2003). In 1993 it was 
E10.5-11.5 mouse limb bud development

(a)

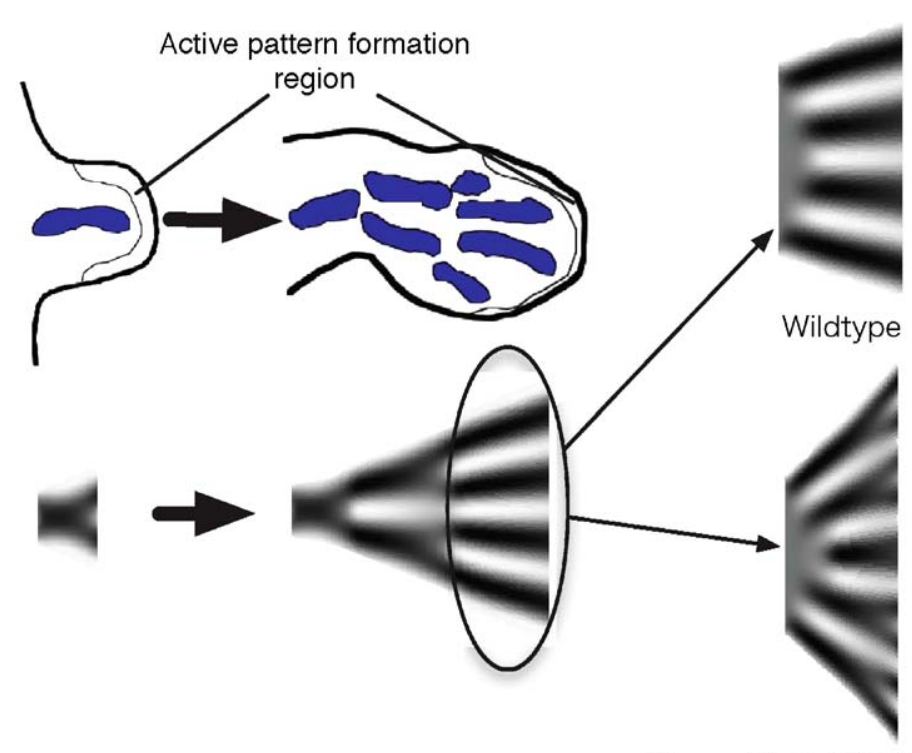

(b)

Doublefoot

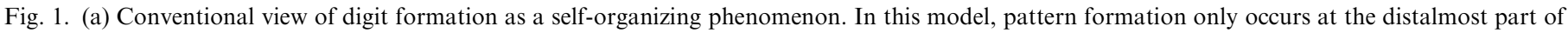

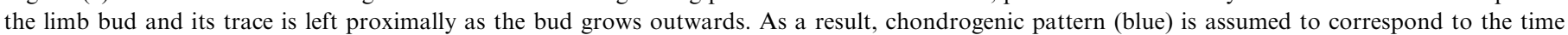

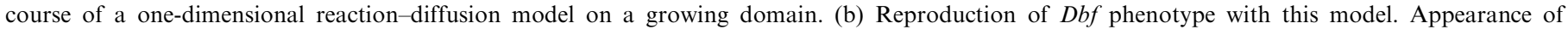
supernumerary digits is observed by increasing growth speed.

shown that digit formation occurs even in the absence of proper Hox code specification (Ros et al., 1994). This observation revitalized research on pattern formation during limb development, and several relevant experiments have recently been carried out in vitro (Miura and Shiota, 2000a, b; Moftah et al., 2002). Recent findings on digit formation in the absence of proper Shh signalling and subsequent Hox genes (Niederreither et al., 2002) further support the idea of self-organized periodic pattern formation during limb development. Current conventional models hypothesize that periodic pattern formation occurs in the distal part of the autopod and leaves traces of the pattern proximally (Hentschel et al., 2004; Chaturvedi et al., 2005); since the autopod is flattened, the pattern can be correlated with the one-dimensional reaction-diffusion model on a growing domain (Fig. 1a).

An example supporting the existence of such a periodic pattern formation mechanism in limb development is provided by polydactylous mutant mice, in which supernumerary digits are formed. For example, the polydactylous mutant mouse Doublefoot (Dbf) has six to eight supernumerary digits without clear identity; in some cases digits arise abruptly from interdigital connective tissue or one digit splits into two distally (Hayes et al., 1998b). Morphologically the autopod is $2-2.5$ times the width of the wrist in the Doublefoot mutant, in contrast to 1.5 times in the wild-type autopod. Overexpansion of the digital plate is associated with ectopic Indian hedgehog (Ihh) expression (Yang et al., 1998) and subsequent acceleration of cell proliferation (Crick et al., 2003). Ectopic Ihh expression is first seen at E10.25 (Crick et al., 2003), by which time the proximal elements have been specified and excessive cell proliferation affects only the carpal/tarsal and digital elements. Therefore we only consider the autopod region of the limb in the simulation. If we increase the domain growth speed at the distal part, the pattern shows additional bifurcation or insertion of digits, which mimics the digit phenotype of the $D b f$ mutant (Fig. 1b).

In the present study, we further investigated the model and the $D b f$ mutant phenotype and found convincing evidence that a one-dimensional Turing reaction-diffusion model on a growing domain is applicable during limb development. Firstly, we show that in $D b f$ mutant limbs, extremely thin digits are sometimes present in the proximal part of the metacarpus/metatarsus, which discontinuously become normal thickness digits more distally. Next we show, by numerical simulations, that this complex mixed mode pattern can be generated by the simplest possible Turing reaction-diffusion system under certain conditions. Then we analytically obtain plausible conditions for mixed mode pattern using matched asymptotics. Next we provide a descriptive explanation of why the mixed mode pattern occurs under certain conditions using the nullclines of the activator kinetics. We then go back to numerical calculations to test the validity of our analytical prediction, and finally show some relevant experimental observations which support the model and discuss possible experimental applications of the model.

\section{Experimental observation: appearance of thin digits in Dbf mutant mice}

Dbf mutant limbs have six to eight digits, and among them we found that there sometimes exist thin digits at 

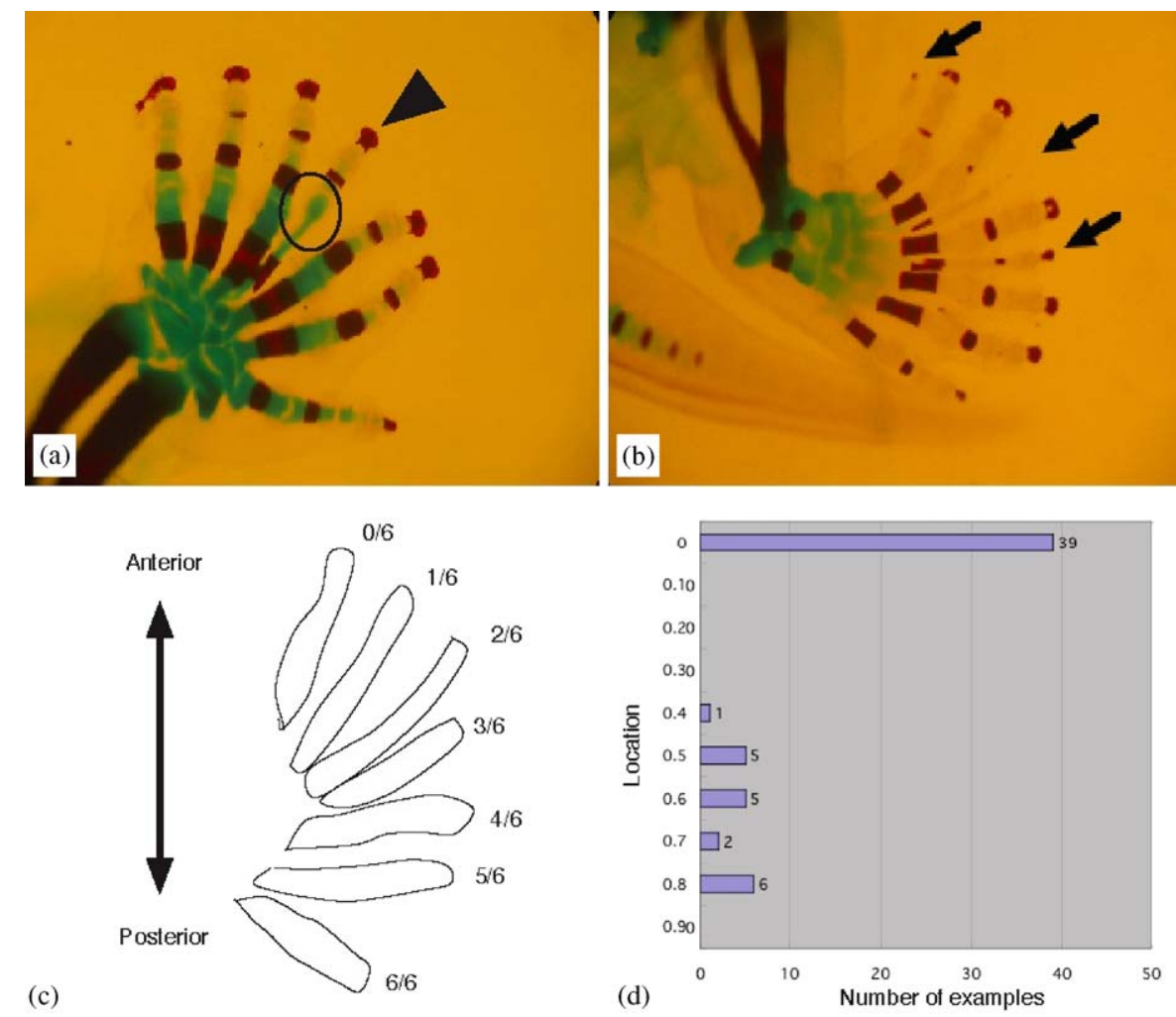

Fig. 2. The shape of mixed-mode patterns. (a) An example of a $D b f$ forelimb. A thin digit (arrowhead) suddenly becomes a digit of normal thickness at its distal part (circle). (b) An example of a $D b f$ hindlimb. Multiple thin digits are observed (arrows). (c) Numbering system for the location of the thin digit. The anteriormost digit was assigned the value 0 and posteriormost digit was assigned the value 1. (d) Distribution of thin digits in $D b f$ limb bud.

variable positions within the digital array (Fig. 2). They were observed in both the fore- and hindlimb, the hindlimb showing a slightly higher frequency (forelimb 22/72, hindlimb 28/72). In some hindlimbs (but not forelimbs), multiple thin digits were observed. Thin skeletal elements were strictly confined to the autopod, never being observed in the proximal parts (zeugopod, stylopod) of the limb. They were most frequently observed in the anteriormost part of the limb but were not confined to that region. To show this, we introduced a numbering system in which the anteriormost digit was assigned the number 0 and the posteriormost digit was assigned the number 1 , and examined the distribution of the location of thin digits (Fig. 2c, d). From this analysis we found that thin digits occurred most frequently in the anteriormost part of the limb. We observed another hot spot of thin digits around location $0.5-0.8$, indicating that in other cases these digits are likely to appear in the posterior half of the limb.

In some cases (4/144) including both fore- and hindlimbs, the thin digit abruptly attains normal thickness distally (Fig. 2a, circle).

\section{Model definition}

We consider the simplest possible form of the Turing reaction-diffusion model which exhibits pattern formation.
The system is defined as follows:

$$
\begin{aligned}
& \frac{\partial u}{\partial t}=f(u, v)+\varepsilon^{2} \gamma^{-1} \Delta u, \\
& \frac{\partial v}{\partial t}=g(u, v)+\gamma^{-1} \Delta v,
\end{aligned}
$$

where

$f(u, v)= \begin{cases}-f_{u} u+f_{v} v-2 f_{u} & (u<-1), \\ f_{u} u+f_{v} v & (-1 \leqslant u \leqslant 1), \\ -f_{u} u+f_{v} v+2 f_{u} & (1<u),\end{cases}$

$g(u, v)=g_{u} u+g_{v} v$.

We choose this piecewise linear model because it is one of the simplest possible models which contains the minimal essential factors for diffusion driven instability. It simply describes the interaction of $u$ and $v$ in linear terms and avoids blow-up of $u$ by adding saturation kinetics when $u$ exceeds a certain threshold. This model is qualitatively similar to the FitzHugh-Nagumo type equations (Murray, 2003), but one advantage of this model is that we can easily obtain approximate analytical solutions using matched asymptotics (described in Section 6.1). Numerical simulation results of the FitzHugh-Nagumo model are qualitatively similar to those presented here (data not shown). 
(a)
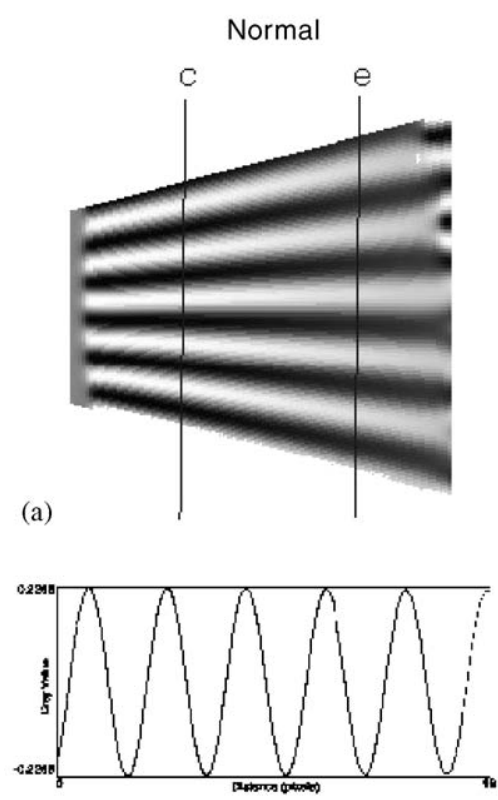

(c)

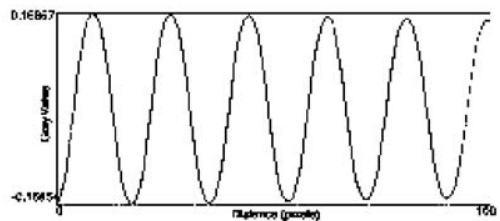

(e)
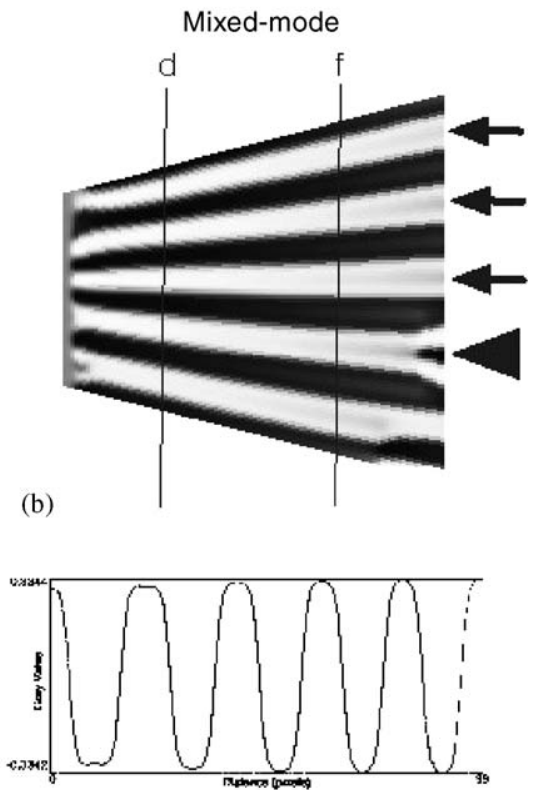

(d)

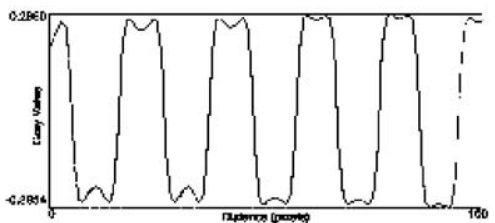

(f)

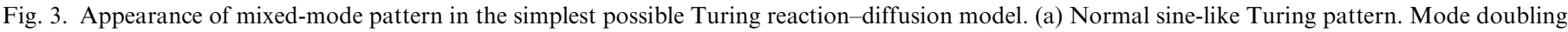

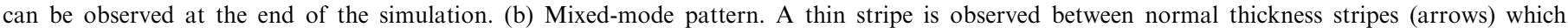

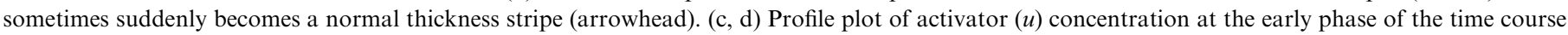

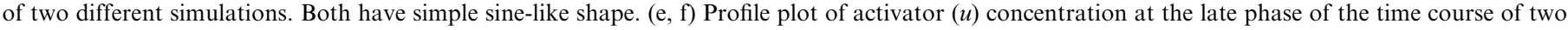
different simulations. Mixed-mode pattern is observed in (f).

Here, $u$ and $v$ represent the concentrations of activator and inhibitor chemicals (morphogens), respectively. We standardize the system so that $u=v=0$ is an equilibrium point. The activator reaction term $f(u, v)$ consists of three linear functions. The second function represents the interaction around the initial $(u$ small) state. The first and third functions represent the interaction after saturation/ depletion of $u$. The inhibitor reaction term $g(u, v)$ consists of a simple linear function. $f_{u}, f_{v}, g_{u}, g_{v}$ are constants and $f_{u}>0, f_{v}<0, g_{u}>0, g_{v}<0 . \gamma$ is a scaling parameter which can capture the domain growth and will increase with time. We use linear growth and assume that the growth process is slow so that the observed pattern is a quasi-steady state. $\varepsilon^{2}$ represents the ratio between activator and inhibitor diffusion coefficient and is less than 1 to satisfy diffusion driven instability conditions (Murray, 2003).

\section{Numerical experiment: thin-digit-like structure appears in some of the reaction-diffusion models on a growing domain}

When the numerical calculation of the Turing reactiondiffusion system on a growing domain was undertaken and the distribution of activator molecule visualized, for some
Table 1

The appearance of mixed-mode pattern is facilitated by domain growth

\begin{tabular}{llc}
\hline & Normal & Mixed mode \\
\hline No growth & 99 & 1 \\
Growing domain & 53 & 47
\end{tabular}

One hundred random Turing reaction-diffusion systems were generated and the patterns they exhibited were analysed. A statistically significant difference is observed between the control (no growth) and growing domain group.

parameter sets the pattern showed a stable thin-digit-like pattern naturally. The thin-digit-like pattern is only seen in $u$ distribution and we could not detect any mixed mode pattern in the distribution of $v$ (data not shown). The pattern was not a transient pattern during peak splitting since peak splitting itself was a rather rapid process and the thin-digit-like pattern persisted much longer than the actual splitting process. In most parameter ranges the thindigit-like pattern was not detected (Fig. 3a, c, e). In some cases, the pattern was at first a simple sine wave-like pattern but gradually evolved to a thin-digit-like pattern 
(Fig. 3b, d, f). We could even detect the appearance of normal digits from thin digits in some cases, which faithfully mimics the example illustrated in Fig. 2a (Fig. 3b, arrowhead). The abrupt appearance of normal-size digits was analysed previously (Crampin et al., 2002a) and is a separate problem, so we concentrate below on the appearance of mixed mode patterns.

To confirm whether the thin-digit-like pattern is observed only on a growing domain, we determined 100 random linear parameter sets $\left(f_{u}, f_{v}, g_{u}, g_{v}, d_{u}, d_{v}\right)$ which satisfy diffusion-driven instability conditions, and undertook numerical calculation to see whether they produce normal sine wave-like patterns or thin-digit-like patterns in fixed domains and in growing domains. In the fixed domain simulations only 1 out of 100 systems showed thin-digitlike pattern while in the growing domains about half of the patterns were of mixed mode type (Table 1). From this result we conclude that domain growth facilitates formation of a thin-digit-like pattern.

\section{Comparison of experimental observation and theoretical results}

Comparing the experimental and simulation results we see excellent agreement between the thin-digit-like pattern sometimes observed in Doublefoot mutant limbs, and those predicted by the Turing reaction-diffusion model under certain conditions. It is difficult to explain this complex pattern change simply by a one-by-one specification with positional information. The molecular cascade which leads to the Doublefoot mutant is well studied, so we are now motivated to determine the conditions under which thindigit-like patterns are generated in the Turing reactiondiffusion model, since this may be correlated to the differences in the molecular cascade occurring in Doublefoot mutant limbs when compared to the wild type limb.

From here on we use the term "mixed mode" to describe this thin-digit-like morphology. "Mode" in this context represents a wavenumber component in the frequency domain and in this pattern one mode (normal thickness digit) coexists with a different mode (thin digit). This is different from the phenomenon of "mode doubling" described by Crampin et al. (2002a) - we use "mixed mode" as a description of a patterned final steady state while "mode doubling" is a description of a dynamic transient process during domain growth.

\section{Analytical derivation of the condition for mixed mode pattern in Turing reaction-diffusion model}

\subsection{Obtaining approximate solutions with a piecewise linear model}

To understand the results of the numerical calculations described above, we undertook a matched asymptotics approach to obtain approximate analytical solutions in the limit of low $d_{u} / d_{v}$ ratio. An overview of the procedure is as follows: at first we assume a specific solution form, which is not a general form but is later confirmed by numerical calculation. Then we construct two solutions, inner and outer, which represent rapidly changing and slowly changing solution parts, respectively, and combine them to obtain an approximate solution (Crampin et al., 2002a).

We suppose $\varepsilon \ll 1$, and consider a plateau-like form solution of $u$ in which there are alternating "high" and "low" states interspersed with transition layers. $v$ should be a smoother simple harmonic wave-like solution because of its high diffusion coefficient. We scale the system so that it contains the span from the centre of the peak to the centre of the neighbouring valley between $0<x<1$. Next, we separately obtain inner and outer solutions, which hold near and far from the transition layer, then match these solutions between separate regions of the piecewise linear function (3). We simplify the analysis by only considering a system which has reverse symmetry, i.e. the system does not change qualitatively under the $(u, v) \rightarrow(-u,-v)$ transformation. In this case the location of the transition layer should be $x=\frac{1}{2}$ and after several approximations (following the method in Crampin et al., 2002b), the solution obtained between $0<x<\frac{1}{2}$ is

$$
\begin{aligned}
u(x)= & 2 \mathrm{e}^{\sqrt{f_{u} \gamma}(x-1 / 2) / \varepsilon}+\frac{2 f_{u} g_{v}}{p} \\
& +\frac{\left.2 f_{v} g_{u} \operatorname{sech}\left(\sqrt{p \gamma / 2 f_{u}}\right) \cosh \left(\sqrt{\left(p \gamma / f_{u}\right.}\right) x\right)}{p}, \\
v(x)= & \frac{2 f_{u} g_{u}}{p}\left(\cosh \left(\sqrt{\frac{p \gamma}{f_{u}}} x\right) \operatorname{sech}\left(\sqrt{\frac{p \gamma}{2 f_{u}}}\right)-1\right),
\end{aligned}
$$

where $p=-\left(f_{u} g_{v}+f_{v} g_{u}\right)>0$. Details of the analysis are described in Appendix C.

Next, we look for the term which contributes to this mixed mode pattern. The first two terms of (4) come from the inner solution and the third term is the contribution of the outer solution. The first term of (4) is an increasing function of $x$ and the second term is constant. Since $p>0$ and $2 f_{v} g_{u} \operatorname{sech}\left(\sqrt{p \gamma / 2 f_{u}}\right)<0$, the third term of (4) is a decreasing function in $x>0$. This term should be a decreasing function in order to generate mixed mode pattern, so this term is responsible for the formation of mixed mode pattern.

\subsection{Intuitive explanation of the mixed-mode pattern}

The mixed-mode pattern comes from the contribution of the outer solution, which can be understood by comparing the spatial distribution of $(u, v)$ (Fig. 4a) and the $u$ nullcline (Fig. $4 \mathrm{~b}$ ). In the outer solution, $\varepsilon^{2} \Delta u$ is small, so $u$ and $v$ satisfy $f(u, v)=0$, and $(u, v)$ should be on the $u$ nullcline (Fig. 4b). Consider a point $a$, which lies between $0<x<\frac{1}{2}$. Then $u$ and $v$ at that point should both be negative. Then consider the $(u, v)$ value of point $b$, which lies slightly to the right of point $a$ (i.e., slightly larger $x$ ) and in $0<x<\frac{1}{2}$, in Fig. 4a. $v$ should increase by this move since the distribution of $v$ is monotonically increasing. Then, $u$ must 
decrease because $(u, v)$ should be on the $u$ nullcline (Fig. 4b). This means that in the outer solution $u$ should decrease as we get close to the transition layer, which results in mixed-mode pattern. Therefore, the upper and lower limits of the activator concentration should result in this type of mixed-mode pattern. This is counteracted by the contribution of the inner solution, so this pattern should be more prominent when $\varepsilon$ is small. When $\gamma$ is large,

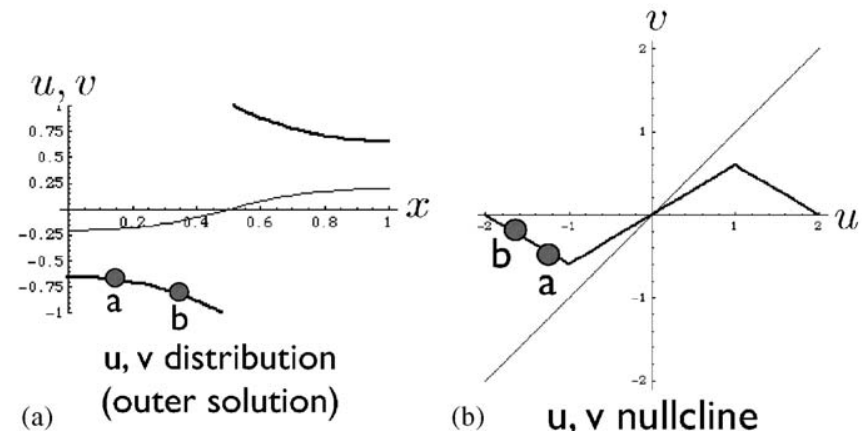

(a)

$$
0=f(u, v)+\gamma^{-1} \epsilon^{2} \Delta u
$$

Fig. 4. Intuitive explanation of the emergence of mixed-mode pattern. Away from the transition layer, the solution should be on the curve $f(u, v)=0$, i.e. on the $u$ nullcline. Since the system is saturated the solution should be on the two lateral branches. Consider the $(u, v)$ value at points $a$ and $b$ on the nullcline. Since the diffusion coefficient of $v$ is large, $v$ should monotonously increase as $x$ increases. Since the solution should be on the nullcline, $u$ has to decrease as $x$ increases, resulting in mixed-mode pattern. the point $a$ has more room to move to the right, which also results in more prominent mixed-mode pattern.

\subsection{Plausible conditions for mixed-mode pattern}

In summary, mixed-mode patterns tend to be generated when

1. $\gamma$ is large and the domain is growing;

2. $\varepsilon$ is small (diffusion coefficients of activator and inhibitor are very different);

3. positive feedback of activator saturates at some point.

\subsection{Verification with numerical simulation}

We verified the above analysis by numerical solution of the equations. A typical result is shown in Fig. 5a. It can be seen that the analytic result is in excellent agreement with the numerical simulation.

Then we examined whether the conditions in Section 6.3 hold. The importance of the first condition in Section 6.3 (large $\gamma$ ) has already been mentioned (see Table 1). To see whether the mixed-mode pattern is likely to be generated with the small $\varepsilon$ condition as predicted in the second condition, we generated 100 Turing reaction-diffusion systems randomly, and divided them into two groupssystems that show mixed-mode pattern and systems that do not. Then we compared the average of $\varepsilon^{2}$ in each group.

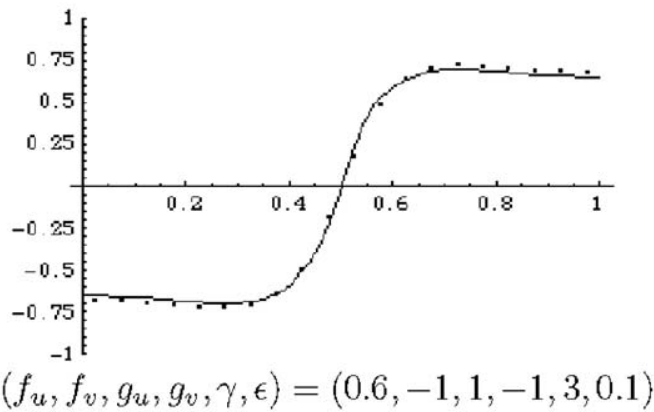

(a)

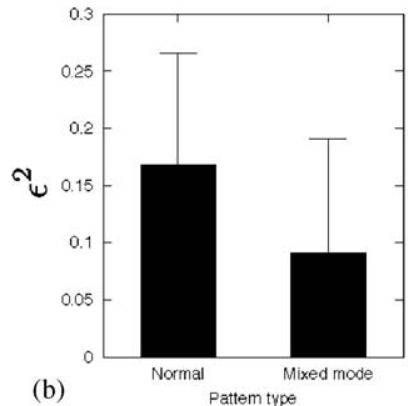

(b)

$$
\begin{aligned}
& \frac{\partial u}{\partial t}=0.6 u-v-u^{3}+d_{u} \Delta u \\
& \frac{\partial v}{\partial t}=1.5 u-2 v+0.25 \Delta v
\end{aligned}
$$
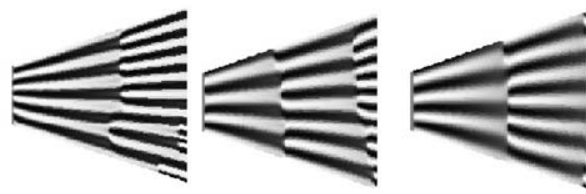

(c)

$d_{u}=0.005$

$d_{u}=0.01$

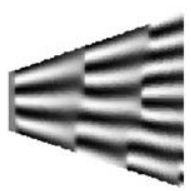

$d_{u}=0.02$

Fig. 5. (a) Comparison of analytical and numerical solutions. Solid line represents analytical solution and dotted line represents numerical simulation. (b) In randomly generated Turing reaction-diffusion systems, the average of $\varepsilon^{2}$ is smaller in the group which generates mixed-mode pattern. (c) Pattern generated when $d_{u}$ is decreased. We observe clearer mixed-mode pattern when $d_{u}$ is smaller. $\left(f_{u}, f_{v}, g_{u}, g_{v}, d_{u}, d_{v}\right)=(0.6,-1,1.5,-2,0.005 \sim 0.02,0.25)$. $t=2000$ 
u profile

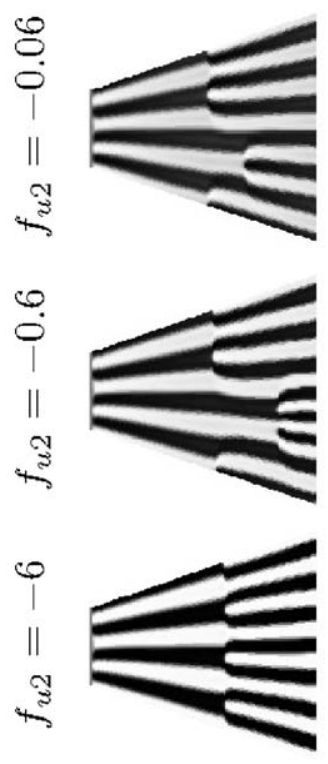

u Nullcline

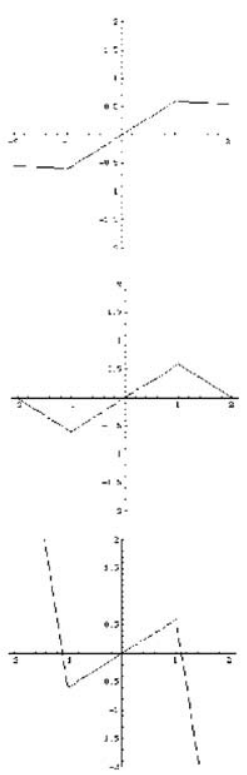

u Profile at $x 2$ growth
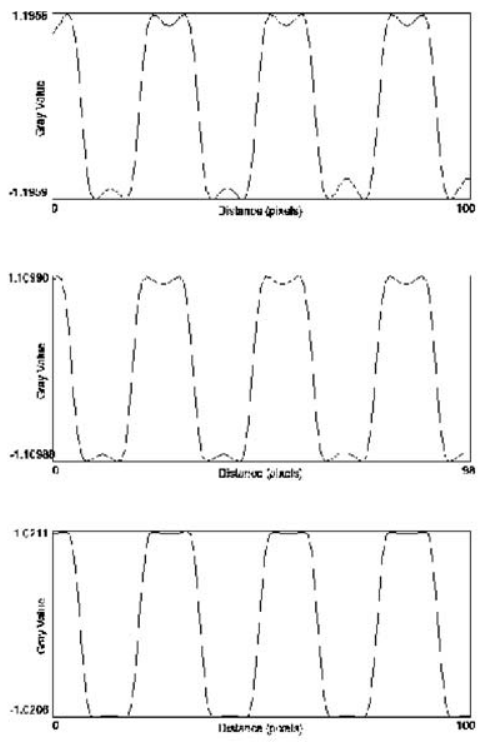

Fig. 6. Saturation kinetics of the reaction-diffusion model affects the appearance of mixed-mode pattern. If we change the gradient of the two saturation branches of the $u$ nullcline, we can modulate the appearance of mixed mode patterns as predicted from the intuitive explanation in Fig. 4.

The average of $\varepsilon^{2}$ is smaller in the group that generates mixed-mode patterns (Fig. 5b). To further confirm this, we focused on one specific reaction-diffusion system and changed $\varepsilon^{2}$. We can observe the appearance of mixed-mode pattern as we decrease $\varepsilon^{2}$, which also confirms the above analysis (Fig. 5c).

Next we examined the third condition-whether the saturation kinetics of the activator reaction term affects the appearance of mixed-mode pattern. From the intuitive explanation using nullclines (Fig. 4) we can predict that if we change the saturated part of the activator kinetics and select a gentler gradient in the nullcline, then the mixedmode pattern will be more apparent. To test this hypothesis, we considered the specific piecewise linear model

$f(u, v)= \begin{cases}f_{u 2}(u+1)-f_{u}+f_{v} v & (u<-1), \\ f_{u} u+f_{v} v & (-1 \leqslant u \leqslant 1), \\ f_{u 2}(u-1)+f_{u}+f_{v} v & (1<u),\end{cases}$

$g(u, v)=g_{u} u+g_{v} v$,

where $\left(f_{u}, f_{v}, g_{u}, g_{v}, d_{u}, d_{v}\right)=(0.6,-1,1.5,-2,0.01,0.25)$, and varied the value of $f_{u 2}$, which corresponds to changing the gradient of the first and third part of the nullcline. When we undertook numerical simulations on a growing domain (domain size $2 \pi$, linear domain growth until the domain doubles in size, $t=2000)$ and increased $\left|f_{u 2}\right|$, the mixed-mode pattern became less apparent, which further validates the above analysis (Fig. 6).

\section{Experimental observation: application of the mixed-mode pattern analysis}

One prediction from this model is that we can observe an inverse pattern - reduced differentiation within thick digits - since the saturation of activator kinetics should occur at both upper and lower limits. We found such an example in actual $D b f$ mutant limbs (Fig. 7). The example illustrated shows a thin undifferentiated area in the middle of the thick digit; the proximal aspect of the digit is of normal thickness, which rules out the possibility that the pattern is generated by fusion of two preexisting digits. This pattern again is very difficult to explain simply by regional specification using positional information gradients, and it further validates the model used in this study.

Another prediction from this model is that since the assumption of the model is quite generic - it only requires the existence of saturation of activator kinetics - this pattern can be observed in other species. Close inspection of the literature reveals existence of numerous such "thindigit" cases. In human polydactyly, thin-digit morphology is sometimes observed (Fig. 7c, arrow), which is frequently described as "rudimentary" or "hypoplastic". In some cases, these supernumerary digits are only thin proximally (Fig. 7c, arrowhead), and the original description runs as follows: "the extra toe consisted of a thin metatarsal, a thicker and better formed proximal phalanx, and a broad distal phalanx" (Temtamy and McKusick, 1978). Another example of the thin-digit phenotype is Daubentonia madagascariensis - a primate generally called aye-aye, in which a thin, elongated middle digit is used to pick out 

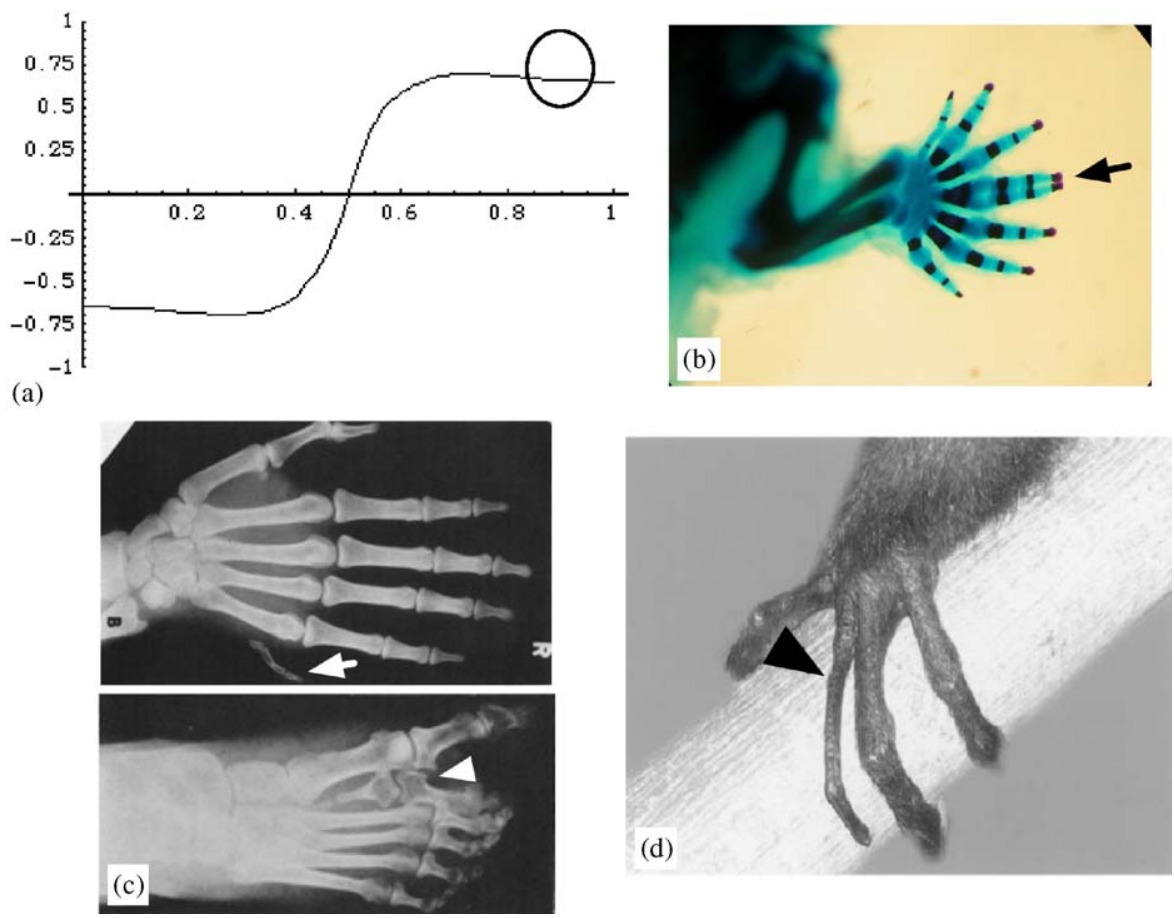

Fig. 7. (a) The mixed-mode activator distribution. The activator reaction should have both upper and lower limits, so there may exist an "inverse" pattern in which a thin undifferentiated area is observed within a thick digit (circle). (b) Actual inverse pattern found in Dbf limb bud. (c) "Thin-digit" morphology observed in human polydactyly (Temtamy and McKusick, 1978). An apparently thin digit is clearly seen in the posteriormost part of the hand (arrow). In some cases the digit starts as a thin digit but becomes of normal thickness distally (arrowhead). (d) An example of a primate hand that shows anatomical"thin-digit" structure (arrowhead) (Krakauer et al., 2002).

insect larvae from small holes in trees (Fig. 7d, arrowhead). It is yet to be elucidated whether this thin digit results from the developmental changes described above or through simple decreased growth at later stages.

\section{Discussion}

One advantage of using the simplest possible model for spatial pattern generation is that the result can be applied to a wide class of models which have the same property. The property we use is (a) diffusion-driven instability, which many mathematical models that generate periodic structure utilize, and (b) saturation of activator kinetics, which is a very natural assumption since in physical systems values never tend to infinity and the activator, which has positive feedback kinetics, should be the first factor to saturate. Most reaction-diffusion models have these properties and hence have an ability to generate mixed-mode pattern.

We believe that mixed-mode patterns arise relatively easily in biological situations since they occur under natural biological conditions. Existence of activator kinetics saturation is obvious as described in the previous paragraph. The small ratio of diffusion coefficients $\left(\varepsilon^{2}\right)$ is consistent with the conditions for diffusion-driven instability, so we will expect the mixed-mode pattern to be frequently observed if periodic pattern is formed. Two examples of skin patterning illustrate this point. In fish skin pigment pattern, where a reaction-diffusion system has been proposed (Kondo and Asai, 1995), mixed-mode pattern is sometimes present (S. Kondo, personal communication). During the development of dermatoglyphics (fingerprint patterns), the epidermal ridges first show a simple periodic structure (primary ridges), then later another furrow is formed within the ridges (secondary ridges), which can be regarded as a mixed-mode pattern (Shauman and Alter, 1976).

We have shown that many of the above experimental observations are consistent with the Turing model. Furthermore, the model analysis we carried out allows us to make experimentally testable predictions. For example, cell culture systems of limb mesenchyme cells can reproduce periodic pattern formation in vitro (Miura and Shiota, 2000a, b), and it may be possible to reproduce domain growth by observing the edge of the cell colony since the colony gradually grows without a barrier and the resulting chondrogenic loci show a radiating pattern. Another tool is TGF $\beta 2$, which is a very good candidate molecule for the activator $u$ (Miura and Shiota, 2000b). We can predict that continuous addition of this molecule to the culture medium results in a change in saturation kinetics and hence a change in the appearance of small structures. The above analysis also implies that $D b f$ mutant mice should have lower activator diffusion coefficient in their 
limbs, which might be experimentally testable utilizing fluorescently labelled TGF $\beta 2$ (Miura and Shiota, 2002), or by monitoring whether Ihh can induce production of certain extracellular matrix components by limb mesenchyme cells, which should modify the diffusion coefficient of the activator molecule.

In our model we could not detect mixed-mode pattern in inhibitor distribution, but this is not true for all systems that generate periodic pattern. For example, Hentschel et al. (2004) used a three-species model and observed mixedmode pattern in both activator and inhibitor distribution. In this case the model equations have nonlinearity in all three species and should be considered separately from our analysis. We should point out that with the present analysis we cannot rule out a higher order mixed-mode contribution to the inhibitor concentration, but our numerical simulations suggest that it would be so small as to be biologically irrelevant.

Some recent studies indicate that transitions in pattern complexity are not solely generated by domain growth. For example, Chaturvedi et al. (2005) argued that in chick, anteroposterior width remains constant during pattern formation. Mathematically, changing diffusion coefficient, overall speed of reaction term, or domain growth are all equivalent, so a combination of these factors may be working in vivo. The mathematical analysis in this paper gives the qualitative result that mixed-mode pattern is more likely to appear when the domain grows more, and this agrees well with the fact that Doublefoot mutant mice sometimes have thin digits.

There are several possible reasons why the thin digit abnormality is not observed between all the digits in the Doublefoot limb bud. One is the existence of noise in such a biological system, which should be much larger than thermal noise in physical systems. Another factor is the faster domain growth in the Doublefoot autopod. From numerical simulations we have an impression that faster domain growth results in uneven distribution of structure. This may be because lower wavenumber components do not have sufficient time to decay completely if the domain growth is fast and this results in regional differences. Another regional difference we observed experimentally is the high frequency of thin digits anteriorly (Fig. 2d), which may be explained by more prolonged and hence greater expansion in the anterior part, which can be seen in Doublefoot limb morphology (Hayes et al., 1998; Crick et al., 2003).

It has been noticed that mixed-mode periodic patterns can appear in reaction-diffusion models on a growing domain (E. Crampin, personal communication) but they have not properly been described or analysed, because their biological importance has not been recognized. This study is one example of how experimental data based on both molecular and morphological observation drive numerical simulation and mathematical analysis to gain insight into mechanisms of pattern formation and then provide evidence for the validity of the model used.

\section{Acknowledgements}

The author is grateful for helpful discussions and comments with Drs. Mikiko Miura, Edmund Crampin, Shigeru Kondo, Atsushi Mochizuki, Axel Rossberg, Tomoyuki Ogawa and Jonathan Bard. This work is supported by Japan Society for the Promotion of Science and BBSRC. We also thank Wiley-Liss Inc. for giving us permission to use Fig. 7c, d.

\section{Appendix A. Skeletal staining of newborn $D b f$ mutant mice}

We used the $D b f$ mutant mouse, which has an autosomal dominant mutation and shows preaxial polydactyly and craniofacial anomaly (Hayes et al., 1998b). Newborn pups were obtained by mating $D b f /+$ mutant mice with $\mathrm{C} 3 \mathrm{H}$ strain wildtype mice. They were fixed overnight with $95 \%$ ethanol; the skin was removed under a dissecting microscope and the pups were stained by alcian blue and alizarin red using standard protocols.

\section{Appendix B. Numerical simulation}

To generate the parameter sets which create periodic pattern within a reasonable period of time, we first generated random parameter sets $\left(f_{u}, f_{v}, g_{u}, g_{v}, d_{u}, d_{v}\right)$ which satisfy the diffusion-driven instability conditions (Murray, 2003). The above condition guarantees the generation of periodic pattern. Next, we rescale the system so that it produces a stable number of periodic structures. The fastest growing wavenumber $k$ in the model is given by

$k=\sqrt{\frac{-d_{u} d_{v}\left(f_{u}-g_{v}\right)+\left(d_{u}+d_{v}\right) \sqrt{-d_{u} d_{v} f_{v} g_{u}}}{d_{u} d_{v}\left(d_{v}-d_{u}\right)}}$,

where $d_{u}=\varepsilon^{2} \gamma^{-1}, d_{v}=\gamma^{-1}$.

Using this equation, we rescale the diffusion coefficients of activator and inhibitor so that the wavenumber within the domain size $2 \pi$ is 5 .

Then we rescale all linear terms to set the growth speed of fastest growing wavenumber (Miura and Maini, 2004), which is

$\lambda_{\max }=\frac{d_{v} f_{u}-d_{u} g_{v}-2 \sqrt{-d_{u} d_{v} f_{v} g_{u}}}{d_{v}-d_{u}}$,

to 0.1 . This makes the system generate stable pattern by $t=100$, which is confirmed by numerical simulations. All the numerical simulations described below are carried out using PowerPC G5 $(1.8 \mathrm{GHz})$. Initial distribution of $u$ and $v$ is constant $(u, v=0)$ with small random noise of order 0.01 . All the simulations were done with a finite difference scheme and zero flux boundary conditions. The number of grid points is 100 and $d t$ was set below $d x^{2} / 2 d_{v}$ to avoid systematic error. The source code of the entire simulations is available from the corresponding author on request.

To detect mixed-mode pattern, first we obtained the pattern after $t=2000$ with or without domain growth. 


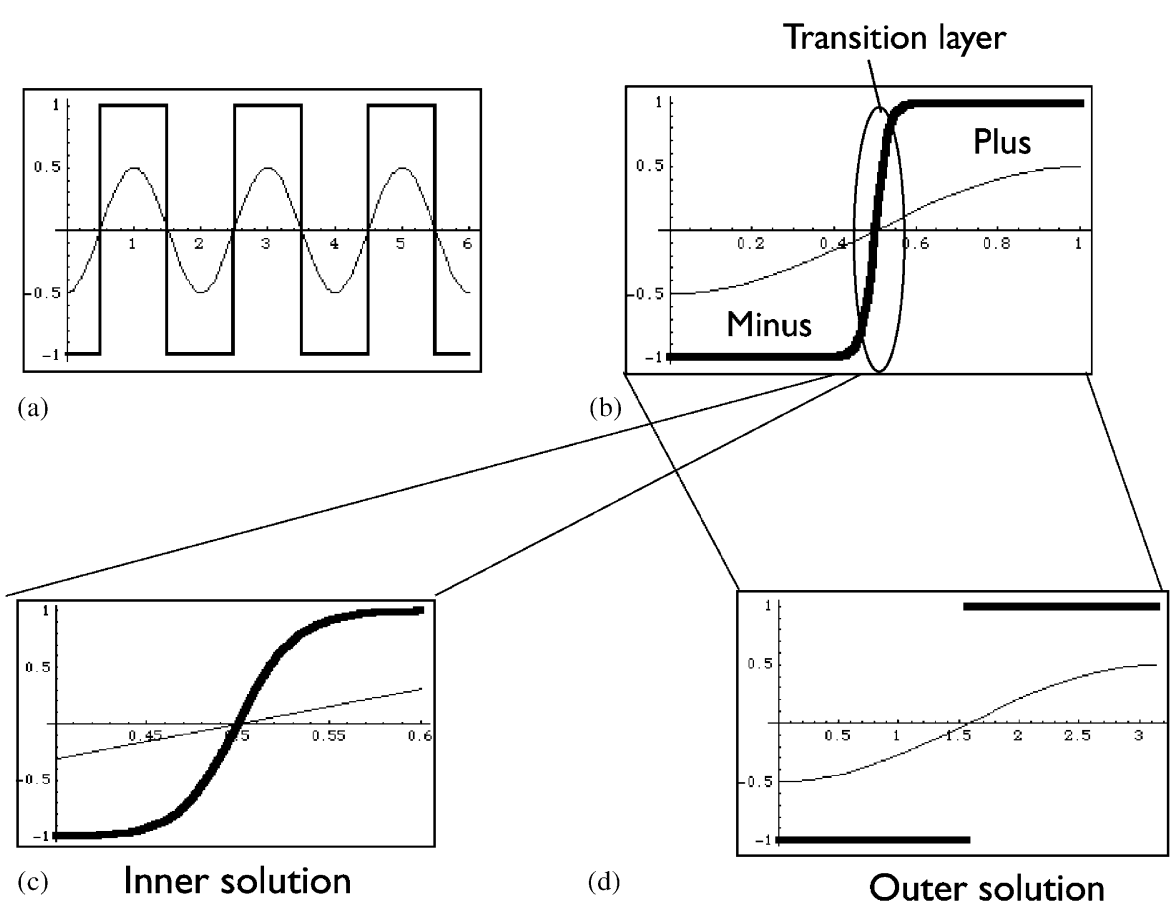

Fig. 8. (a) Assumption of solution form. Alternating plus and minus states are separated by transition layers. (b) We construct approximate solution of half the period - from the centre of a valley to the centre of a neighbouring peak. (c) The outer solution holds away from the transition layer. (d) The inner solution holds near the transition layer.

Then we counted the number of points that cross the $u=0$ line and those which change the gradient from positive to negative or vice versa. If the latter is 1.5 times more than the former we define the pattern as a mixed-mode pattern.

\section{Appendix C. Derivation of approximate analytical solution}

\section{C.1. Definition of inner and outer subsystems}

The original analysis was done by Crampin et al. (2002a) with asymmetry of reaction term considered, but in this case we use a simplified analysis with reverse symmetry since we concentrate on the mechanism of generation of mixed-mode pattern.

We obtain steady state solutions of the system defined in (3)

$0=f(u, v)+\varepsilon^{2} \gamma^{-1} \Delta u$,

$0=g(u, v)+\gamma^{-1} \Delta v$,

where

$f(u, v)= \begin{cases}-f_{u} u+f_{v} v-2 f_{u} & (u<-1 \text { : region } 1), \\ f_{u} u+f_{v} v & (-1 \leqslant u \leqslant 1 \text { : region } 2), \\ -f_{u} u+f_{v} v+2 f_{u} & (1<u \text { : region } 3),\end{cases}$

$g(u, v)=g_{u} u+g_{v} v$.

We divide the reaction term of $u$ into three regions according to $u$ value. We define $u<-1$ as region 1 , $-1 \leqslant u \leqslant 1$ as region $2,1<u$ as region 3 , respectively.
To obtain the steady state solution of system (10), we suppose $\varepsilon \ll 1$, and consider a plateau-like form solution of $u$ in which there are alternating "high" and "low" states interspersed with transition layers (Fig. 8a). $v$ should be a smoother simple harmonic wave-like solution because of its high diffusion coefficient. We scale the system so that it contains the span from the centre of the valley to the centre of the neighbouring peak between $0<x<1$. The location of the transition layer should be $x=\frac{1}{2}$ because the system has reverse symmetry, i.e. the shape of peak and inverted valley should be the same.

We obtain the outer solution, which holds away from the transition layer (Fig. 8c), and the inner solution, which holds near the transition layer and has different spatial scale (Fig. 8d). The outer solution must satisfy

$0=f(u, v)$,

$0=g(u, v)+\gamma \Delta u$.

in the limit $\varepsilon \rightarrow 0$.

The inner solution holds near the transition layer, so we define another spatial parameter $\xi$

$$
\xi=\left(x-\frac{1}{2}\right) / \varepsilon \text {. }
$$

Then the inner subsystem becomes

$$
\begin{aligned}
& 0=f(u, v)+\gamma \frac{\partial^{2} u}{\partial \xi^{2}}, \\
& 0=g(u, v)+\gamma \varepsilon^{-2} \frac{\partial^{2} v}{\partial \xi^{2}} .
\end{aligned}
$$


In the $\varepsilon \rightarrow 0$ limit, this becomes

$0=f(u, v)+\gamma \frac{\partial^{2} u}{\partial \xi^{2}}$,

$0=\gamma \frac{\partial^{2} v}{\partial \xi^{2}}$

\section{C.2. Obtaining outer solutions}

The peak and valley portions of the outer solutions (Fig. 8c) should be in regions 3 and 1, respectively, so appropriate equations are (13), (14) and the relevant terms from (11). We define the outer solutions in regions 1 and 3 as $\left(u_{o 1}, v_{o 1}\right)$ and $\left(u_{o 3}, v_{o 3}\right)$, respectively. At first, using (13) we can obtain the relationship between $u(x)$ and $v(x)$ :

$u(x)=\frac{-2 f_{u}+f_{v} v(x)}{f_{u}}$.

Substituting (18) into (14) yields

$\frac{\partial^{2} v}{\partial x^{2}}+\frac{f_{v} g_{u}+f_{u} g_{v}}{f_{u}} \gamma v=2 g_{u} \gamma$

and we can obtain the distribution of $v$ in region 1 as

$v_{o 1}(x)=\frac{2 f_{u} g_{u}}{f_{v} g_{u}+f_{u} g_{v}}+2 C_{0} \cosh \left(\frac{x \sqrt{-\left(f_{v} g_{u}+f_{u} g_{v}\right) \gamma}}{\sqrt{f_{u}}}\right)$,

where $C_{0}$ is a constant of integration. $C_{0}$ can be obtained by the reverse symmetry condition: $v_{o 1}(x)$ must be 0 at the transition layer $x=\frac{1}{2}$. Therefore

$C_{0}=-\frac{f_{u} g_{u} \operatorname{sech}\left(\sqrt{-\left(f_{v} g_{u}+f_{u} g_{v}\right) \gamma} / 2 \sqrt{f_{u}}\right)}{f_{v} g_{u}+f_{u} g_{v}}$.

We then obtain $u_{o 1}$ using (18). Summarizing, the outer solution in region 1 is $\left(u_{i 3}, v_{i 3}\right)$, respectively. We need a solution where the $u$ value changes abruptly at the transition layer but $v$ does not. Therefore, we set the $v$ value for the inner subsystem as constant. The $v$ value at $x=\frac{1}{2}$ should be 0 because of the reverse symmetry of the whole system, so (17) becomes

$0=f(u, 0)+\gamma^{-1} \frac{\partial^{2} u}{\partial \xi^{2}}$

For region 1, the solution is

$u_{i 1}(\xi)=-2+C_{1} \mathrm{e}^{\sqrt{f_{u} \gamma \xi}}+C_{2} \mathrm{e}^{-\sqrt{f_{u} \gamma \xi}}$,

where $C_{1}$ and $C_{2}$ are constants of integration. We need a solution which connects to the outer solution as $\xi \rightarrow-\infty$, so $C_{2}$ should be 0 because otherwise $u_{i 1}$ goes to infinity.

From reverse symmetry of the whole system, we can obtain the solution in region 3 as follows:

$u_{i 3}(\xi)=-u_{i 1}(-\xi)$.

For region 2, the solution is

$u_{i 2}(\xi)=C_{3} \operatorname{Sin}\left(\sqrt{f_{u} \gamma} \xi\right)$.

The resulting form of $u(x)$ is somewhat cumbersome, but if we neglect the inner solution in region 2 and consider the solutions in regions 1 and 3 only, we do not change the matching condition for the leading order calculation and retain good approximation to the numerical solution (Crampin et al., 2002a).

From the condition $u_{i 3}(0)=u_{i 1}(0)$, we find that $C_{1}=2$ and then the resulting inner solution in terms of $x$ is

$u_{i 1}(x)=-2+2 \mathrm{e}^{\sqrt{f_{u} \gamma}(x-1 / 2) / \varepsilon}$.

$u_{i 3}$ can be obtained by the reverse symmetry condition.

$u_{i 3}=-u_{i 1}(1-x)$.

\section{C.4. Composite solution}

To obtain the composite solution, we substitute the constant term of the inner solution (30) into the outer

$u_{o 1}(x)=-\frac{2 f_{u} g_{v}}{f_{v} g_{u}+f_{u} g_{v}}-\frac{2 f_{v} g_{u} \cosh \left(x \sqrt{-\left(f_{v} g_{u}+f_{u} g_{v}\right) \gamma} / \sqrt{f_{u}}\right) \operatorname{sech}\left(\sqrt{-\left(f_{v} g_{u}+f_{u} g_{v}\right) \gamma} / \sqrt{2 f_{u}}\right)}{f_{v} g_{u}+f_{u} g_{v}}$,

$v_{o 1}(x)=\frac{2 f_{u} g_{u}}{f_{v} g_{u}+f_{u} g_{v}}-\frac{2 f_{u} g_{u} \cosh \left(x \sqrt{-\left(f_{v} g_{u}+f_{u} g_{v}\right) \gamma} / \sqrt{f_{u}}\right) \operatorname{sech}\left(\sqrt{-\left(f_{v} g_{u}+f_{u} g_{v}\right) \gamma} / \sqrt{2 f_{u}}\right)}{f_{v} g_{u}+f_{u} g_{v}}$.

The outer solutions in region 3 can be derived using the reverse symmetry of the system:

$u_{o 3}(x)=-u_{o 1}(1-x)$,

$v_{o 3}(x)=-v_{o 1}(1-x)$.

\section{C.3. Obtaining inner solutions}

The inner solution should use regions $1-3$, so we define inner solutions for these regions as $\left(u_{i 1}, v_{i 1}\right),\left(u_{i 2}, v_{i 2}\right)$, solution (23) since the constant term is the value when the inner solution is away from the transition layer $(\xi \rightarrow \infty)$. The resulting composite solution is shown in the main text (4).

\section{References}

Bard, J.B., 1981. J. Theor. Biol. 93 (2), 363.

Chaturvedi, R., Huang, C., Kazmierczak, B., Schneider, T., Izaguirre, J., Glimm, T., Hentschel, H., Newman, S., Glazier, J., Alber, M., 2005. Interface (R. Soc. London) 2, 237. 
Crampin, E.J., Gaffney, E.A., Maini, P.K., 2002a. J. Math. Biol. 44 (2), 107.

Crampin, E.J., Hackborn, W.W., Maini, P.K., 2002b. Bull. Math. Biol. 64 (4), 747

Crick, A.P., Babbs, C., Brown, J.M., Morriss-Kay, G.M., 2003. J. Anat. 202 (1), 21.

Gierer, A., Meinhardt, H., 1972. Kybernetik 12 (1), 30.

Gilbert, S.F., 2004. Developmental Biology. Sinauer, Massachusettes.

Hayes, C., Brown, J.M., Lyon, M.F., Morriss-Kay, G.M., 1998 a. Development 125 (3), 351.

Hayes, C., Lyon, M.F., Morriss-Kay, G.M., 1998b. J. Anat. 193 (Pt 1), 81.

Hentschel, H.G.E., Glimm, T., Glazier, J.A., Newman, S.A., 2004. Proc. R. Soc. London B Biol. Sci. 271 (1549), 1713.

Jung, H.S., Francis-West, P.H., Widelitz, R.B., Jiang, T.X., Ting-Berreth, S., Tickle, C., Wolpert, L., Chuong, C.M., 1998. Dev. Biol. 196 (1), 11 Kondo, S., Asai, R., 1995. Nature 376, 765.

Krakauer, E., Lemelin, P., Schmitt, D., 2002. Am. J. Primatol. 57 (3), 105. Maini, P.K., Solursh, M., 1991. Int. Rev. Cytol. 129, 91.

Miura, T., Maini, P.K., 2004. Bull. Math. Biol. 66 (4), 627.

Miura, T., Shiota, K., 2000a. Anat. Rec. 258 (1), 100.

Miura, T., Shiota, K., 2000b. Dev. Dyn. 217 (3), 241.

Miura, T., Shiota, K., 2002. Mech. Dev. 116 (1-2), 29.
Moftah, M.Z., Downie, S.A., Bronstein, N.B., Mezentseva, N., Pu, J., Maher, P.A., Newman, S.A., 2002. Dev. Biol. 249 (2), 270.

Murray, J.D., 2003. Mathematical Biology, third ed. Springer, Berlin.

Newman, S.A., Frisch, H.L., 1979. Science 205, 662.

Newman, S.A., Frisch, H.L., Percus, J.K., 1988. J. Theor. Biol. 134, 183.

Niederreither, K., Fraulob, V., Garnier, J.M., Chambon, P., Dolle, P., 2002. Mech. Dev. 110 (1-2), 165.

Oster, G.F., Murray, J.D., Harris, A.K., 1983. J. Embryol. Exp. Morphol. $78,83$.

Othmer, H.G., 1986. J. Theor. Biol. 121 (4), 505.

Ros, M.A., Lyons, G.E., Mackem, S., Fallon, J.F., 1994. Dev. Biol. 166 (1), 59.

Shauman, B., Alter, M., 1976. Dermatoglyphics in Medical Disorders, first ed. Springer, New York.

Temtamy, S., McKusick, V., 1978. The Genetics of Hand Malformations. Birth Defects Original Article Series, vol. 14. National Foundation, New York.

Turing, A.M., 1952. Philos. Trans. R. Soc. B 237, 37.

Wolpert, L., 1998. Principles of Development. Oxford University Press, New York.

Yang, Y., Guillot, P., Boyd, Y., Lyon, M.F., McMahon, A.P., 1998. Development 125 (16), 3123. 